\title{
DINÁMICA DEL OXÍGENO EN EL HIPOLIMNION DE UN EMBALSE NUEVO Y OLIGOTRÓFICO DE ALTA MONTAÑA PROVISTO DE SISTEMA DE BOMBEO
}

\author{
A. Palau Ibars \\ Institut d'Investigació d'Alta Muntanya. Univcrsitat de Barcelona. Vall d'Aran, LLEIDA. España \\ Palabras clave: oxígeno, hipolimnion, profundidad de estabilización, aguas entrantes.
}

\begin{abstract}
DYNAMIC OF OXYGEN CONCENTRATION IN THE HYPOLIMNION OF A RECENT AND OLIGOTROPHIC HIGH MOUNTAIN PUMPING RESERVOIR
\end{abstract}

The Baserca Reservoir is located in the Central Pyrenees (Lleida. NE-Spain) at $1434 \mathrm{~m}$ a.s.l. This reservoir had some external and internal aspects with consequences on the general distribution of oxygen content, and especially for the hypolimnion case. The dam was closed in 1984 and the reservoir has been studied since the spring of 1985; so it was a recent reservoir under the effects of the first mineralisation processes of sediments. It is also a reservoir with a high water turnover, and connected with a old damed lake (Llauset Lake: $2192 \mathrm{~m}$ a.s.l.) through a reversible hydroelectric power station (Moralets) which takes out or inject water from/to the bottom of reservoir. At the same time, the climatology of its area, its thermal structure and the internal organization of flow often makes a preferential distribution in depth of incoming stream waters.

Taking into account the whole of this aspects, the purpose of this paper is to explain the hypolimnetic oxygen budget of reservoir for the period which it was sampled. The results shows a great oxygen consumption; however it was not detected with the routine procedure of sampling.

\section{INTRODUCCIÓN}

Baserca es un embalse nuevo situado en la vertiente meridional de los Pirineos Centrales, en la cabecera del río Noguera Ribagorzana. Se trata de un reservorio de agua relativamente pequeño, con un volumen máximo de $22,20 \mathrm{hm}^{3}$, una configuración semejante a la de un rectángulo de dimensiones medias $2,05 \mathrm{~km}$ de longitud y $0,42 \mathrm{~km}$ de anchura, y una profundidad media de $24 \mathrm{~m}$. Este embalse presenta varios aspectos que hacían interesante el conocer la dinámica del oxígeno en su hipolimnion.

En primer lugar Baserca, en el momento del estudio era un embalse reciente y a pesar de ello no se detectó en ninguna ocasión anoxia en el fondo. Sí que se detectaron en cambio discontinuidades

Limnctica. $7:$ 175-182 (1991)

(C) Asociación Española de Limnología, Madrid, Spain verticales de varias variables (ph, conductividad, oxígeno) que hacían intuir el mantenimiento de una circulación horizontal de las aguas entrantes. Por otro lado, Baserca era y es un embalse conectado a un antiguo lago ahora represado, Llauset, mediante una central hidroeléctrica reversible (turbina/bomba), capaz de movilizar volúmenes importantes (hasta $30 \mathrm{~m}^{7} . \mathrm{s}^{1}$ ) de aguas embalsadas profundas tanto del lago como del embalse. Esta central reversible se puso por primera vez en funcionamiento en el mes de agosto de 1985.

Todas las entradas y salidas fluyentes de agua alrededor del embalse están aforadas, lo que permite llevar a cabo estimaciones de balances con bastante precisión. Por otro lado la red de drenaje que alimenta el embalse es importante $(122 \mathrm{hm}$ ' año ' en el año estudiado) y se encuentra nada o muy poco alterada, lo que determina una tasa de renovación alta y al mismo tiempo condiciona el carácter oligotrófico del embalse. Es por último, 
un embalse de alta montaña y por tanto sometido a una climatología rigurosa.

La finalidad de este artículo es exponer e interpretar el balance hipolimnético del oxígeno en el embalse. Teniendo en cuenta que los datos utilizados no pertenecen a un estudio específico sobre este tema concreto, interesa más analizar la forma de obtención de los resultados que los valores concretos, si bien éstos presentan el interés del orden de magnitud al que corresponden y del sentido de su evolución.

\section{MATERIAL Y MÉTODOS}

La figura 1 muestra la localización orientativa del embalse de Baserca, así como otros aspectos de interés. Los datos base de este artículo corresponden al seguimiento, aproximadamente quincenal, de tres perfiles verticales situados sobre el eje del embalse (cola, centro y presa) cuyas profundidades máximas eran de $17 \mathrm{~m}, 32 \mathrm{~m}$ y $69 \mathrm{~m}$ y su separación de la presa de 1740 m, 860 m y 65 m respectivamente. La duración total del estudio al

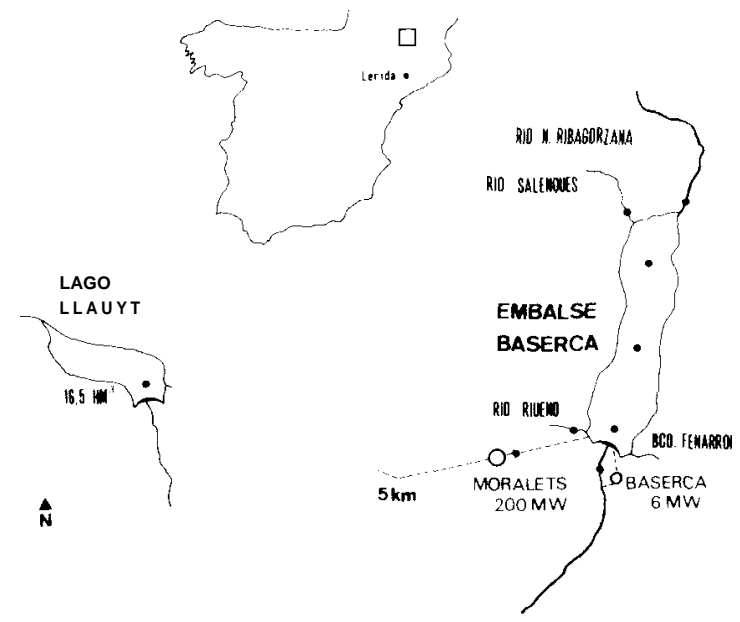

Figura 1.- Localización geográfica del embalse de Baserca. de los puntos de muestreo (•) y de la disposición de los ríos confluyentes. En la misma figura se muestran algunos datos generales como referencias.

General plane of location of the study area, showing some general characteristics, the situation of sampling stations $(\bullet)$, the incoming streams and the relationship between the rescrvoir and the Llauset Lake. que corresponden los datos (PALAU, 1988) fue de 14 meses, desde junio de 1985 hasta agosto de 1986, durante los cuales se llevaron a cabo 26 campanas de muestreo de las que en este artículo se utilizan las 11 primeras comprendidas entre el 15 de junio de 1985 y el 26 de noviembre del mismo ano.

Las concentraciones de oxígeno se determinaron metro a metro hasta los $30 \mathrm{~m}$ que era la longitud máxima del cable del oxímetro utilizado. A partir de esta profundidad, sólo existente en el punto más próximo a la presa, las determinaciones se llevaban a cabo cada $10 \mathrm{~m}$ sobre el agua recogida con botellas Van Dorn, hasta alcanzar los $60 \mathrm{~m}$ de profundidad, dependiendo de la cota del embalse. Los valores se obtuvieron mediante un oxímetro Syland 610. Paralelamente también se recogieron muestras de los principales cursos de agua que confluyen en el embalse, de las aguas a la salida y de las intercambiadas con el lago de Llauset.

Para el contrastado de los datos, así como el control de calibrado del oxímetro se aplicó periódicamente el método Winkler a muestras duplicadas. Las diferencias obtenidas nunca excedieron de 0,3 ppm $\mathrm{O}_{2}$, siendo el valor más habitual el de 0,1 ppm $\mathrm{O}_{2}$.

En la figura 2 se muestra el planteamiento de base de los cálculos realizados. Como ya se ha dicho, no se llevó a cabo un muestreo específico sobre el hipolimnion del embalse, por lo que la falta de datos sobre la concentración de oxígeno a diferentes cotas, se ha estimado a partir de interpolaciones y extrapolaciones lineales de los datos existentes, como aproximación. El error que se pueda cometer con tal planteamiento puede admitirse ya que, por un lado tienen más peso específico los valores de los volúmenes de agua implicados que las concentraciones de oxígeno y, por otro lado el posible error afectaría sólo a las partidas del balance de oxígeno dependientes directamente de la concentración de oxígeno en el hipolimnion que, como se verá, intervienen mucho menos en los resultados que las dependientes de los flujos de agua entrantes.

Para la estimación del balance de oxígeno el hipolimnion se subdividió en dos estratos, desde la cota 1.381 (nivel inferior de las tomas de la presa) hasta la 1.366 (nivel inferior de los desagües de fondo) y desde ésta hasta la 1.358 (nivel mínimo 
del embalse). El contenido de oxígeno en ambos estratos se dedujo de la concentración media (estimada por ponderación con la profundidad de las concentraciones obtenidas o calculadas) y del volumen correspondiente.

Los aportes de oxígeno al hipolimnion se dividieron, para cada mes, en dos tipos: 1) aportes externos debidos a la entrada de los ríos y de las aguas turbinadas desde el lago de Llauset (ambos estimados a partir de los volúmenes mensuales aportados y sus respectivas concentraciones medias de oxígeno); 2) aportes internos debidos a las aguas provenientes del compartimento superior al hipolimnion y que entraron en éste para compensar los volúmenes perdidos por filtraciones a través de la presa y por la apertura de los desagües de fondo (su estimación se ha hecho en base al volumen de agua intercambiado y a la concentración media de oxígeno en la cota límite superior del hipolimnion).

Las pérdidas de oxígeno del hipolimnion también se han dividido en dos tipos. 1) Pérdidas exteriores debidas a las salidas de agua desde el hipolimnion hacia el exterior del embalse (filtraciones a través de la presa y apertura de los desagües de fondo). Su cálculo se ha hecho a partir de los volúmenes de agua extraídos y de la concentración media de oxígeno en todo el hipolimnion para el caso de las filtraciones. La estimación de la pérdida de oxígeno del hipolimnion a través de los desagües de fondo es complicada ya que por su volumen, debió afectar a todo el hipolimnion, a los compartimentos superiores y a las aguas procedentes de los ríos o de Llauset que alcanzaban el fondo en aquel momento (de hecho, el contenido de oxígeno en el hipolimnion aumentó un 7,4\% en octubre con la apertura de los desagües). En este caso, la concentración media de oxígeno utilizada en los cálculos se dedujo de la que presentaba el volumen de agua equivalente al desalojado, en el interior del embalse, desde la cota 1.395 m s.n.m. hasta la cota de los desagües de fondo (1366 m s.n.m.). 2) Pérdidas interiores debidas a los volúmenes de agua que pasaron desde el hipolimnion al compartimento inmediatamente superior para compensar las entradas de agua de ríos y las turbinadas desde Llauset. Su estimación se hizo a partir del volumen de agua intercambiado y de la concentración media de oxígeno en el estrato superior del hipolimnion.
El transporte vertical de oxígeno por difusión en la capa límite superior del hipolimnion se ha calculado, mes a mes, a partir del gradiente de oxígeno existente entre las concentraciones de la cota 1381 y las inmediatamente superiores (figura 2) sobre el que se ha aplicado un coeficiente de difusión turbulenta para sustancias disueltas (K,) estimado a partir de la expresión propuesta por SNOdgrass \& O'Melia (1975) como una función exponencial de la altura media de la capa de agua considerada ( $\mathrm{z}$, en metros): $\mathrm{K}_{\mathrm{z}}=0,00682 \mathrm{z}^{1.18}$.

El valor resultante varía entre $0,0919 \mathrm{~m}^{\prime} \mathrm{d}^{-1} \mathrm{y}$ $0,0194 \mathrm{~m}^{\prime} \mathrm{d}^{-1}$ es del mismo orden de magnitud que los encontrados por CoRnET \& RigLer (1987) en un estudio dedicado al transporte vertical de oxígeno en el hipolimnion de lagos.

\section{RESULTADOS}

Los tres principales cursos de agua que aportaban sus aguas al embalse (ríos Noguera Ribagorzana, Salenques y Riueno), por su escasa profun-

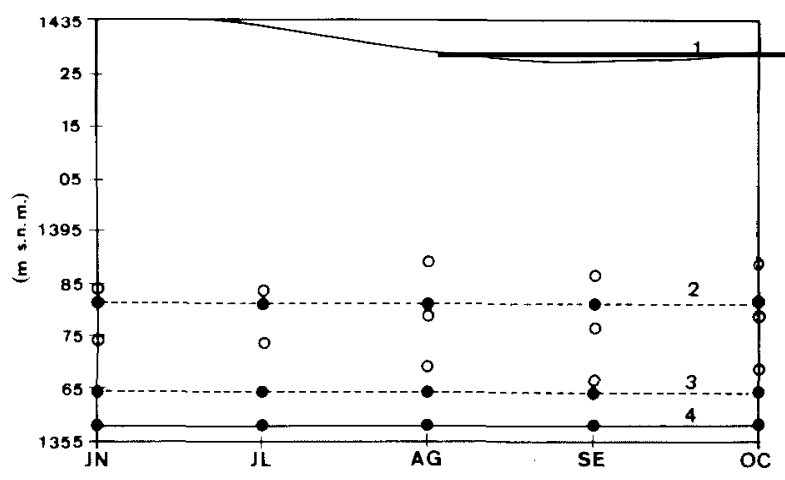

Figura 2.- Definición de los límites del hipolimnion, de los dos estratos considerados (2-3 y 3-4) y distribución de los puntos de muestreo medidos (O) e interpolados o extrapolados (•) utilizados en el cálculo del balance de oxígeno. (1) Variación del nivel del agua embalsada; (2) Límite superior del hipolimnion y cota de las tomas de salida; (3) Cota de los desagües de fondo; (4) Cota mínima del embalse.

Definition of hypolimnctic boundaries and the two considered layers (2-3 and 3-4). Distribution of sampling locations measurcd ( 0 ) and interpolated or extrapolated ( $\bullet$ ) which were used in thc oxygen budgct. (1) Fluctuation of level of damed water; (2) Upper boundary of hypolimnion and level of water turbine outlet; (3) Level of bottom outlet; (4) Level of the bottom of reservoir. 
Tabla 1.- Profundidades a las que se encontraron las concentraciones de oxígeno más altas respecto a la profundidad de los perfiles realizados en los tres puntos de muestreo, entre junio y noviembre de 1985. En los casos en los que se daban concentraciones iguales a diferentes niveles, la tabla recoge las que se encontraron a mayor profundidad.

Depths where the maximal concentration of oxygen was recorded in the three vertical profiles, from juny to november 1985 . When in one of this profiles the maximal concentration of oxygen were the same at differents depths, the value selected and showed in the tahle was the deep.

\begin{tabular}{|c|c|c|c|c|c|c|}
\hline & \multicolumn{2}{|c|}{ Cola del embalse } & \multicolumn{2}{|c|}{ Centro del embalse } & \multicolumn{2}{|c|}{ Presa del embalse } \\
\hline & $\begin{array}{l}\text { prof. } \\
\text { máx. }\end{array}$ & $\begin{array}{c}\text { prof. } \\
{\left[\mathrm{O}_{2}\right] \text { max }}\end{array}$ & $\begin{array}{l}\text { prof. } \\
\text { máx. }\end{array}$ & $\begin{array}{c}\text { prof. } \\
{\left[\mathrm{O}_{2}\right] \text { máx. }}\end{array}$ & $\begin{array}{l}\text { prof. } \\
\text { máx. }\end{array}$ & $\begin{array}{c}\text { prof. } \\
{\left[\mathrm{O}_{2}\right] \text { máx. }}\end{array}$ \\
\hline 16106185 & $16 \mathrm{~m}$ & $11 \mathrm{~m}$ & $30 \mathrm{~m}$ & $6 \mathrm{~m}$ & $60 \mathrm{~m}$ & $50 \mathrm{~m}$ \\
\hline $28 / 06 / 85$ & $17 \mathrm{~m}$ & $16 \mathrm{~m}$ & $30 \mathrm{~m}$ & $14 \mathrm{~m}$ & $60 \mathrm{~m}$ & $14 \mathrm{~m}$ \\
\hline 15107185 & $17 \mathrm{~m}$ & $14 \mathrm{~m}$ & $30 \mathrm{~m}$ & $17 \mathrm{~m}$ & $60 \mathrm{~m}$ & $18 \mathrm{~m}$ \\
\hline 29107185 & $17 \mathrm{~m}$ & $13 \mathrm{~m}$ & $30 \mathrm{~m}$ & $10 \mathrm{~m}$ & $60 \mathrm{~m}$ & $13 \mathrm{~m}$ \\
\hline $12 / 08 / 85$ & $17 \mathrm{~m}$ & $9 \mathrm{~m}$ & $30 \mathrm{~m}$ & $6 \mathrm{~m}$ & $60 \mathrm{~m}$ & $40 \mathrm{~m}$ \\
\hline $29 / 08 / 85$ & $15 \mathrm{~m}$ & $6 \mathrm{~m}$ & $30 \mathrm{~m}$ & $8 \mathrm{~m}$ & $60 \mathrm{~m}$ & $9 \mathrm{~m}$ \\
\hline 30109185 & $9 \mathrm{~m}$ & $4 \mathrm{~m}$ & $24 \mathrm{~m}$ & $3 \mathrm{~m}$ & $60 \mathrm{~m}$ & $5 \mathrm{~m}$ \\
\hline 10110185 & $11 \mathrm{~m}$ & $7 \mathrm{~m}$ & $26 \mathrm{~m}$ & $26 \mathrm{~m}$ & $60 \mathrm{~m}$ & $40 \mathrm{~m}$ \\
\hline 23110185 & $12 \mathrm{~m}$ & $12 \mathrm{~m}$ & $27 \mathrm{~m}$ & Y m & $60 \mathrm{~m}$ & $50 \mathrm{~m}$ \\
\hline $06 / 11185$ & $13 \mathrm{~m}$ & $13 \mathrm{~m}$ & $28 \mathrm{~m}$ & $8 \mathrm{~m}$ & $60 \mathrm{~m}$ & $29 \mathrm{~m}$ \\
\hline $26111 / 85$ & $13 \mathrm{~m}$ & $13 \mathrm{~m}$ & $28 \mathrm{~m}$ & $11 \mathrm{~m}$ & $60 \mathrm{~m}$ & $60 \mathrm{~m}$ \\
\hline
\end{tabular}

didad y su régimen turbulento, no presentaron grandes desviaciones del $100 \%$ de saturación en oxígeno en ninguna época del ano. Estos tres ríos que son los que se muestrearon, representan en conjunto el $94,5 \%$ del flujo entrante dentro el balance hídrico anual del embalse.

Debido a la temperatura relativa (densidad) de las aguas embalsadas respecto a las entrantes y a la diferente inercia térmica de ambas frente a los cambios climatológicos estacionales, las aguas de los ríos se estabilizaban durante gran parte del año en profundidad en el interior del embalse, dando lugar a discontinuidades verticales apreciables en cuanto al oxígeno, no justificables en muchos casos por una mayor actividad fotosintética o por el efecto de la temperatura como se ha señalado para los lagos oligotróficos (CORNETT \& R $\mathrm{R}$ GLER. 1987). La tabla 1 muestra de un modo sencillo las profundidades inferiores en las que se registraron los máximos de oxígeno a lo largo de las 11 campanas de muestreo consideradas, en relación con la profundidad de los perfiles. Un aspecto destacable de esta misma tabla es la notable consistencia de los datos en el plano horizontal, lo que permitió intuir que incluso la circulación de los ríos que entraban por la cola del embalse (Noguera Ribagorzana y Salenques), en muchos casos se proyectaban hasta el perfil más próximo a la presa.
Esta aparente estabilización subsuperficial preferente de las aguas entrantes junto con la elevada tasa de renovación bruta del embalse, estimada en 11,5 año $^{-1}$ a partir de la relación flujo/ volumen, introducían un factor de variabilidad notable que incluso hacía incoherentes algunas interpretaciones de parámetros físico-químicos a lo largo del ciclo anual. Por esta razón se llevó a cabo un estudio del embalse en base a compartimentos verticales establecidos a partir de criterios morfométricos y de la disposición de las tomas de agua en la presa (PALAU, 1988). En esencia, la compartimentación consistió en la definición de 5 compartimento verticales sobre los que, a partir de la temperatura (densidad) de las aguas entrantes, se deducía su nivel de estabilización preferente en uno u otro compartimento. Por otro lado, como cada compartimento era, por definición, de volumen constante excepto el más superior que variaba según la cota del embalse, se podían estimar directamente los intercambios de agua entre compartimento .En definitiva se disponía de una estructura que, para un compartimento dado, permitía conocer de un modo aproximado las entradas y salidas de agua bien de tipo directo (entrada de ríos, turbinados del lago y del embalse), bien de tipo indirecto (volúmenes de agua desplazados desde otros compartimentos). Dentro de esta compartimentación el hipolimnion, de acuer- 
Tabla 2.- Datos obtenidos para el cálculo del balance de oxígeno en el hipolimnion del embalse de Baserca Obtained values for the estimation of the oxygen hudget in the hypolimnion of reservoir of Baserca.

\begin{tabular}{|c|c|c|c|c|c|c|}
\hline $\begin{array}{l}\text { Estratos } \\
\text { (m.s.n.m.) }\end{array}$ & $\begin{array}{c}\text { Volumen } \\
\left(m^{3}\right)\end{array}$ & $\begin{array}{c}/ \mathrm{O}_{2} / \mathrm{med} \\
(\mathrm{mg} / \mathrm{l}) \\
28 / 06 / 85\end{array}$ & $\begin{array}{c}\mathrm{O}_{2} \text { total } \\
(\mathrm{Kg})\end{array}$ & $\begin{array}{c}\mathrm{/O} / \mathrm{med} \\
(\mathrm{mg} / \mathrm{l}) \\
23 / 10 / 85\end{array}$ & $\begin{array}{c}\mathrm{O}_{2} \text { total } \\
(\mathrm{kg})\end{array}$ & $\begin{array}{c}\text { Diferencia } \\
(\mathrm{kg})\end{array}$ \\
\hline $1381-1366$ & 597.466 & 7.6 & 4.541 & 5,0 & 2.987 & \\
\hline \multirow[t]{2}{*}{$1366-1358$} & 54.648 & 5,1 & 279 & 1,2 & 66 & \\
\hline & 652.114 & & 4.820 & & 3.053 & 1.767 \\
\hline
\end{tabular}

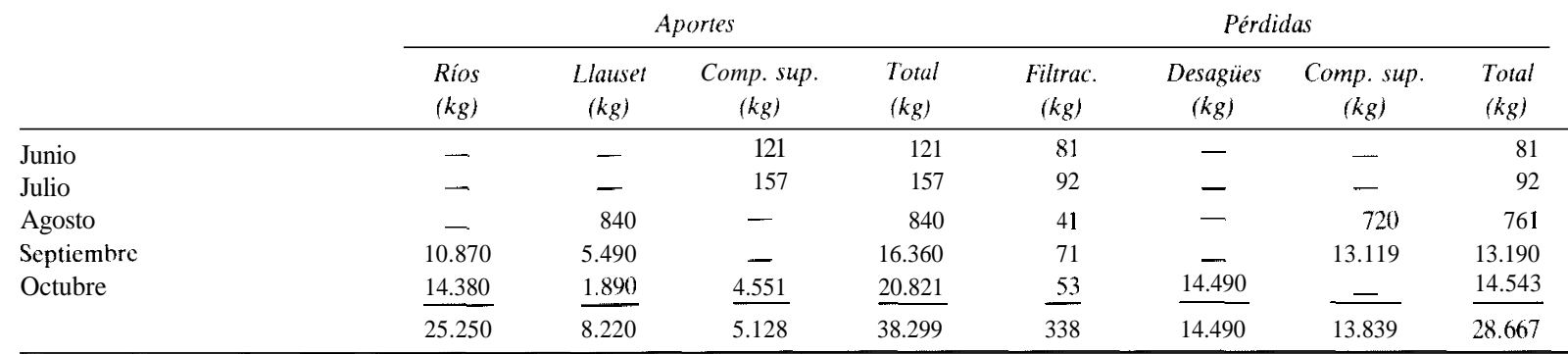

do con la baja fluctuación vertical de la termoclina estimada a lo largo del tiempo, cifrable en $\pm 4 \mathrm{~m}$ alrededor de la cota de las tomas de salida del embalse, se consideró como el compartimento más profundo.

La termoclina empezó a detectarse a finales de junio y se mantuvo hasta mediados de noviembre, si bien a los efectos de este artículo sólo se consideró el período de estratificación más marcada, entre finales de junio y finales de octubre.

La tabla 2 muestra los datos de partida para el cálculo del balance de oxígeno en el hipolimnion. De acuerdo con la temperatura (densidad) de las aguas entrantes y la compartimentación del embalse, se dedujo que el agua turbinada desde el lago de Llauset alcanzaba el hipolimnion durante los meses de agosto, septiembre y octubre, y que las aguas de los ríos Salenques y Riueno lo hacían en septiembre y octubre. Por otro lado, se conocía el volumen desalojado por los desagües de fondo en unas pruebas realizadas en octubre, que aunque provocó un descenso transitorio de la termoclina debido a su magnitud (unas 3,6 veces el volumen del hipolimnion), no llegó a romperla (o no se detectó) posiblemente por la propia estabilización en profundidad, durante el mismo mes, de las aguas más frías turbinadas desde Llauset y de las procedentes de los ríos que debieron compensar gran parte del volumen desalojado.
Si al contenido de oxígeno en el hipolimnion de finales de junio se le aplican los aportes y pérdidas totales y se le resta el contenido residual final del mes de octubre, se obtiene una cantidad de oxígeno de $11399 \mathrm{~kg}$ (frente a los $1767 \mathrm{~kg}$ que se deducen de la diferencia directa entre ambas épocas del año). Este consumo hipolimnético de oxígeno para el período considerado (117 días) y la superficie del hipolimnion $\left(79622 \mathrm{~m}^{2}\right)$, equivale a

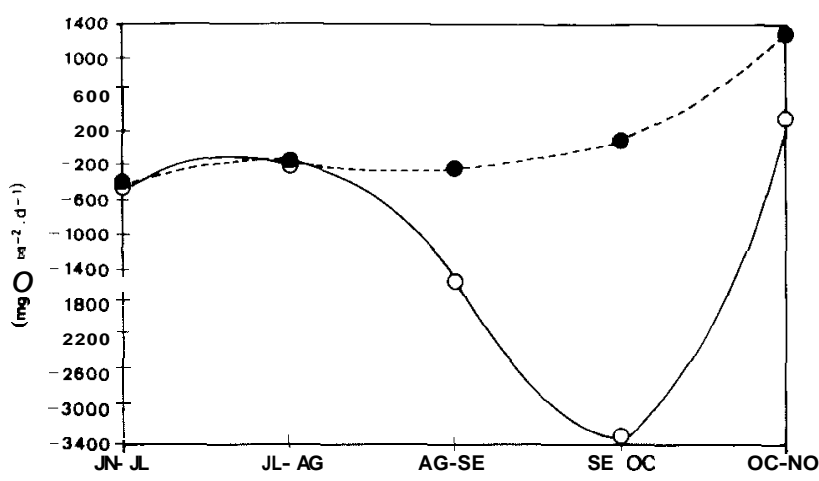

Figura 3.- Evolución temporal de la tasa de consumo de oxígeno por unidad de superficie y tiempo entre los diferentes meses, desde el inicio de la estratificación hasta el momento pre. vio a la mezcla vertical.

Temporal evolution of oxygen depletion rate per surface and time units over the stratification period. 
Tabla 3.- Variación del oxígeno disuelto con la profundidad en el límite superior del hipolimnion. valores de los coeficientes de difusión y aporte (positivo) o pérdida (negativo) de oxígeno hacia/desde el hipolimnion.

Variation of oxygen concentration in depth $\left(\left[\mathrm{O}_{2}\right] / z\right)$ in the uppcr boundary of the hypolimnion vertical eddy diffusivity coeffieient $\left(\mathrm{K}_{2}\right)$, and the vertical transport of oxygen resultant to (positive) or from (negative) the hypolimnion (T.V.).

\begin{tabular}{|c|c|c|c|c|c|}
\hline & Junio & Julio & Agosto & Septiembre & Octubre \\
\hline$\left[\mathrm{O}_{2}\right] / \mathrm{z}\left(\mathrm{mg} \mathrm{O}_{2} \cdot \mathrm{m}^{-3} \cdot \mathrm{m}^{-1}\right)$ & +26.5 & +282 & +112 & -154 & +29 \\
\hline$K_{z}\left(m^{2} \cdot d^{-1}\right)$ & 0.0194 & 0.0215 & 0,0432 & 0,0451 & 0,0919 \\
\hline T.V. ( $\left.\mathrm{mg} \mathrm{O}_{2} \cdot \mathrm{m}^{-2} \cdot \mathrm{d}^{-1}\right)$ & +5.1 & +6.0 & +4.8 & -6.9 & $+1,8$ \\
\hline
\end{tabular}

$1,2 \mathrm{~g} \mathrm{O}_{2} \mathrm{~m}^{2} \cdot \mathrm{d}^{-1}\left(4,6 \mathrm{mmol} \mathrm{O}_{2} \cdot \mathrm{m}^{3} \cdot \mathrm{d}^{-1}\right)$ que es un valor muy superior al estimado por VIDAL (1972) para el embalse hipereutrófico de Sau (río Ter, $0,1333 \mathrm{~g} \mathrm{O}_{2} \cdot \mathrm{m}^{-2} \cdot \mathrm{d}^{1}$ ) o al estimado por Planas (1973) de 0,94 $\mathrm{mmol} \mathrm{O}_{2} \cdot \mathrm{m}^{3}$.d ${ }^{\mathrm{t}}$ para el lago de Bañolas considerado oligotrófico y de dimensiones similares al embalse. Estos dos datos de referencia son mucho más comparables a los obtenidos a partir de la diferencia directa del contenido de oxígeno entre junio y octubre $\left(0,19 \mathrm{~g} \mathrm{O}_{2} \cdot \mathrm{m}^{2} \cdot \mathrm{d}^{-1}\right.$ ó $0,72 \mathrm{mmol} \mathrm{O}_{2} \cdot \mathrm{m}^{3} \cdot \mathrm{d}^{-1}$ ).

Los $1,2 \mathrm{~g} \mathrm{O}_{2} \cdot \mathrm{m}^{2} \cdot \mathrm{d}^{-1}$ representan el consumo medio de oxígeno a lo largo del período considerado. La evolución temporal de este valor mes a mes se ha representado en la figura 3 junto con la referida al resultado del balance hipolimnético de oxígeno sin tener en cuenta los aportes y las pérdidas $\left(0,19 \mathrm{~g} \mathrm{O}_{2} \cdot \mathrm{m}^{2} \cdot \mathrm{d}^{-1}\right)$. Para definir más en el tiempo el sentido de la evolución de las dos tasas de consumo medio de oxígeno, se han añadido en la misma figura los datos correspondientes al mes de noviembre, cuando las aguas turbinadas desde Llauset y las procedentes de los tres ríos entrantes se estabilizaban en el hipolimnion. Las diferencias entre uno y otro caso son muy claras. Así, si no se tienen en cuenta las entradas y las salidas de oxígeno en el hipolimnion, la tasa de consumo de oxígeno disminuye (hacia valores positivos) de un modo aproximadamente lineal entre finales de junio y finales de octubre, mientras que teniendo en cuenta los datos de la tabla 2, la tasa de consumo aumenta de un modo exponencial (hacia valores cada vez más negativos) para el mismo período. Por otro lado, del primer caso (evolución lineal), se deduce que el consumo hipolimnético de oxígeno se compensó a partir del mes de octubre; mientras que en el segundo caso (evolución exponencial) esto no ocurrió hasta el mes de noviembre, momento previo ya a la mezcla vertical.
La importancia del transporte vertical de oxígeno por difusión en la capa límite superior del hipolimnion, como una partida más del balance, puede evaluarse a partir de los datos que se presentan en la tabla 3 donde los valores negativos se interpretan como pérdidas de oxígeno desde el hipolimnion. El valor máximo se dió en el mes de julio y más concretamente entre finales de julio y mediados de agosto (339 $\mathrm{mg} \mathrm{O} \cdot \mathrm{m}^{-3} \cdot \mathrm{m}^{-1}$ ) como aporte de oxígeno hacia el hipolimnion. La suma de los valores absolutos medios mensuales que se muestran en la tabla no representa más que un $2 \%$ de la tasa media de cambio a lo largo del tiempo, con lo que no afecta significativamente a la disminución de oxígeno hipolimnético. Por otro lado, en el momento de plena estratificación (finales de agosto y septiembre) el hipolimnion tendió a exportar oxígeno por difusión hacia el compartimento inmediatamente superior. Estos dos últimos aspectos se han observado también en lagos (CORNETT \& RigLER, 1987), aún cuando éstos no tienen una componente horizontal en su termoclina, debida al flujo de salida, comparable a la del embalse de Baserca.

\section{DISCUSIÓN}

Los resultados encontrados ponen de manifiesto la importancia de tener en cuenta los aportes y las pérdidas de oxígeno derivados de la circulación interna de ríos y sistemas afluentes o efluentes, en los balances hipolimnéticos de oxígeno, especialmente en embalses sometidos a una tasa de renovación alta. En el mismo sentido, la compartimentación se intuye como una estructura útil para la deducción, al menos orientativa, de los intercambios verticales de volúmenes de agua. 
Las primeras fases de consolidación de un embalse suponen un aumento más o menos importante y reversible del grado de eutrofia, destacando la anoxia en profundidad que tal circunstancia lleva implícita (VIDAL, 1969, 1972 y 1976). En el embalse de Baserca, por el contrario y a pesar de que no se limpió de vegetación la zona a inundar, no se siguió este patrón de anoxia pero el consumo hipolimnético de oxígeno fue ciertamente muy alto, incluso superior al de los lagos más eutróficos según los rangos propuestos por HuTCHINSON (1975), siendo sólo comparable al de sistemas hipereutróficos con una recarga inicial de oxígeno notable en la mezcla primaveral o bien, como en el caso de Baserca, a sistemas con constantes aportes adicionales de oxígeno en profundidad que mantienen concentraciones por encima de la anoxia y que si no se tienen en cuenta dan una estima del consumo de oxígeno muy inferior.

En el embalse de Baserca el tiempo de residencia del agua en el hipolimnion no llegó a ser excesivamente alto, debido a su volumen y sobre todo a la disposición en profundidad de las aguas de los ríos y de las turbinadas desde el lago de Llauset, de los volúmenes desalojados a través de los desagües de fondo y de los intercambiados entre compartimentos. La importancia de estos aspectos queda de manifiesto en la capacidad de exportación de oxigeno por difusión desde el hipolimnion en plena estratificación (septiembre) así como por el aumento del contenido de oxígeno detectado a finales de octubre aún bajo una marcada termoclina.

La evolución temporal del contenido de oxígeno disuelto en el hipolimnion, parece ser por tanto más dependiente de la organización interna del embalse que de sus propias características tróficas (oligotrófico). Esta dependencia se vió reforzada por la climatología de la zona y por la existencia de una termoclina coincidente con la cota de las tomas de salida del embalse que pudo actuar como una doble barrera desde el epilimnion hacia el hipolimnion, en primer lugar por el propio gradiente de densidad y en segundo lugar favoreciendo que gran parte de la materia orgánica epilimnética en sedimentación fuera retirada del embalse a través de las tomas de salida antes de alcanzar el hipolimnion disminuyendo así la carga orgánica a mineralizar en el fondo. Este aspecto posiblemente es cxtrapolable a otros embalses.
Admitiendo que la productividad del embalse es baja y que el hipolimnion ve disminuido el aporte de materia orgánica epilimnética por lo ya comentado, el consumo hipolimnético de oxígeno a lo largo del tiempo puede considerarse atribuible en su mayor parte, a la mineralización de los sedimentos recientes que pudo tener lugar ininterrumpidamente a expensas del continuado aporte de oxígeno. Está claro que un balance del oxígeno hipolimnético se acaba cuando se alcanza la condición de anoxia y que ello depende de la productividad del sistema y de la relación entre la superficie de los sedimentos en contacto con el agua $(S$,$) y el volumen hipolimnético \left(V_{h}\right)$. Sin embargo, en un sistema oligotrófico de las características de Baserca, con aportes de oxígeno netos importantes y continuados, si la causa principal de consumo (mineralización del sedimento en este caso) se mantiene sin compensarse, la tasa de consumo por unidad de superficie y tiempo puede ser muy alta, mayor incluso que la de un hipotético sistema más eutrófico, sin aportes y con un hipolimnion de dimensiones similares $\left(\mathrm{S} / \mathrm{V}_{\mathrm{h}}\right)$, en el que el oxígeno se agote más rápidamente. Bajo este planteamiento la relación entre consumo de oxígeno en el hipolimnion y productividad del embalse, pierde sentido.

Queda por explicar la causa del incremento exponencial que sigue la tasa de consumo de oxígeno mostrada en la figura 2. En condiciones normales la demanda de oxígeno por parte del sedimento debería ser más o menos constante en el tiempo e incluso con tendencia a disminuir a medida que se va formando lo que Wetzel (1981) llama la microzona oxidada, que actúa limitando la demanda de oxígeno por parte de los sedimentos. En el caso de Baserca debe tenerse en cuenta que el sedimento está formado por antiguos suelos (pastos) con una acumulación de materia orgánica notable en el horizonte superior (rankers) que podría suponerse inicialmente más o menos inmovilizada a causa de la citada microzona superficial oxidada. La entrada de aguas al hipolimnion, especialmente las turbinadas (inyectadas) desde el lago de Llauset, al mismo tiempo que aportaron oxígeno, pudieron ser causa de la alteración por turbulencia de esta microzona dando lugar a una resuspensión continuada de sedimento, o en general a un aumento difícilmente evaluable de la superficie de materia orgánica en contacto con el 
agua, con el correspondiente incremento de la demanda de oxígeno y de las tasas de consumo mensuales, bajo condiciones aerobias mantenidas por el conjunto de aportes de agua por el fondo. Si se observa la figura 2 , se ve como el incremento exponencial ocurre a partir de agosto, precisamente el mes en el que se empezó a turbinar aguas desde el lago de Llauset hacia el hipolimnion del embalse.

\section{Bibliografía}

Cornett, R.J. \& F.H. Rigler, 1987. Vertical Transport of Oxygen into the Hypolimnion of Lakes. Can. J. Fish. Aquat. Sci.. 44: 852-858.

Hul-chinson, G.E.. 1975. A treatise ori Limnology, I. Geography, Physics and Chemistry. John Wiley \& Sons. Inc. New York. 540 pp.
PAl AU. A.. 1988. El cmbalse de Baserca (Pirineos Centrales, Lérida). Estudio limnológico de un embalse nuevo de alta montaña con bombeo. Tesis Doctoral. Universidad de Barcelona.

Pi ANAS, D.. 1973. Composición, ciclo y productividad del lago de Banyoles. Oecologia aquatica, 1: 3-106.

Snodgrass, D.W. \& C.R. O'Mel.IA, 1975. Predictive model for phosphorus in lakes. Environ. Sci. Techtiol., 9: 937-944

VIDAI . A.. 1969. Evolution d'un lac de barrage dans le NE de l'Espagne pendant les quatre premières années de service. Verh. Internat. Verein. Limnol., 17: 191-200.

VIDAL. A.. 1972. Dinámica del hipolimnion anóxico en el embalse de Sau (Barcelona). Piririeos, 105: 129-169.

Vidal. A.. 1976. Eutrofización del embalse de Sau en el transcurso de sus primeros arios (1963-1972). Publicaciones de la Confederación Hidrográfica del Pirineo Oriental, Secc. 1." Explotación Aguas Ter. Cardedeu (Barcelona). $43 \mathrm{pp}$.

Wetzel, R.G., 1981. Limnología. Ed. Omega. Barcelona. $679 \mathrm{pp}$. 